\title{
Childhood interstitial lung disease due to compound heterozygous mutations of the ABCA3 gene
}

\author{
Kok Joo Chan ${ }^{1}$, Meow-Keong Thong ${ }^{1}$, Anna Nathan ${ }^{2}$, Surendran Thavagnanam ${ }^{2}$, Yusnita \\ Yakob $^{3}$, and Chin Gan ${ }^{4}$ \\ ${ }^{1}$ University of Malaya Medical Centre \\ ${ }^{2}$ University of Malaya - City Campus \\ ${ }^{3}$ Institute for Medical Research \\ ${ }^{4}$ University of Malaya Faculty of Medicine
}

August 20, 2020

\begin{abstract}
Introduction: Mutations in adenosine triphosphate-binding cassette transporter A3 (ABCA3) (OMIM:601615) gene was the commonest genetic cause for severe neonatal respiratory distress syndrome (RDS) and childhood interstitial lung disease (chILD). Most literature review has been from patients of Caucasian heritage. Case Description: We report a case of a term male newborn of South East Asian heritage that developed severe respiratory distress soon after birth, and diagnosis was made on clinical, radiological and genetic basis without lung biopsy. He was found to have compound heterozygous variants for the adenosine triphosphate-binding cassette transporter A3 (ABCA3) gene: a novel c.3364G>A (p.Glu1122Lys) variant and previously reported pathogenic c. $737 \mathrm{C}>\mathrm{T}$ (p.Pro246Leu). The variant c.3364G $>$ A (rs1233043384) was not previously identified in clinical/disease databases such as ClinVar and The Human Gene Mutation Database. Further analysis and bioinformatics study indicated this variant was likely pathogenic. Parental genetic studies revealed parents had one mutation each. Conclusion: Newborns with unexplained respiratory distress syndrome (RDS) and pulmonary surfactant deficiency should have genetic sequencing study. This will be vital for early diagnosis, disease prognostication, treatment planning such lung transplantation, genetic counseling and provide the molecular basis for prenatal diagnosis and pre-implantation genetic diagnosis. It is important to raise awareness of this condition in the Southeast Asia region. Further larger population-based studies are required to determine the frequency of this mutation in our population. ABCA3 deficiency should be considered in term babies who develop severe respiratory distress syndrome in this region.
\end{abstract}

\section{INTRODUCTION}

Childhood Interstitial Lung Disease (chILD) is a heterogenous group of rare chronic respiratory disorders in childhood that features remodeling of the lung interstitium and distal airspaces resulting in poor gas exchange and presence of diffuse parenchymal infiltrates on radiography or computer tomography ${ }^{1,2}$. The incidence of chILD is estimated at 0.13-16.2 cases/100,000 children per year ${ }^{2}$. A number of genes, including $A B C A 3, S F T P B, S F T P C$ are implicated in this condition. ABCA3(ATP-Binding Cassette, Subfamily A, Member 3) which is expressed in alveolar type II cells and localized to the membrane of lamellar bodies is involved in pulmonary surfactant production ${ }^{3,4}$. Pathogenic variants of the $A B C A 3$ gene have been reported in various pulmonary phenotypes in terms of age of presentation, disease severity and progression ${ }^{5,6}$, and has been attributed to surfactant insufficiency or surfactant metabolism dysfunction pulmonary 3 (SMDP3) (OMIM \#601615) $)^{7.8}$. Although ABCA3 gene mutations have been reported in different ethnicities, however the variants in Asian children may be different and thus making prognostication challenging ${ }^{9}$. We report a case of a full-term Southeast Asian boy with compound heterozygous $A B C A 3$ variants which are classified as 'likely pathogenic' as the cause of his fatal respiratory disease. 


\section{INFORMED CONSENT}

Informed consent has been taken from both parents regarding research article publication.

\section{CASE REPORT}

Our patient was a term boy, born at 39 weeks gestation via spontaneous vertex delivery with a birthweight of $2.99 \mathrm{~kg}$. Antenatally, apart from a single episode of maternal H1N1 infection during the $2^{\text {nd }}$ trimester, there were no other complications. The father (aged 41 years) and mother (aged 35 years) were unrelated and had no significant medical or family history. They had a 6-year-old daughter who was well.

The patient was vigorous at birth with an Apgar score of 7 and 8 at $1^{\text {st }}$ and $5^{\text {th }}$ minute, respectively. At the $1^{\text {st }}$ hour of life, he developed respiratory distress with tachypnoea and grunting, requiring noninvasive ventilation (NIV) and intravenous antibiotics for suspected congenital pneumonia. He continued to deteriorate and required invasive ventilation by day 2. The initial chest X-ray at day 1 of life (Figure 1) showed left retrocardiac and upper lobe haziness. Echocardiogram showed a structurally normal heart, and excluded ductal-dependent cardiac lesions. He was given two doses of endotracheal surfactant with transient improvement. In addition, antimicrobial coverage was widened, a short course of intravenous dexamethasone was given, and oral spironolactone and hydrochlorothiazide were added for suspected acute respiratory distress syndrome.

The patient was extubated on day 9 of life to NIV but required reintubation at day 11 of life for worsening respiratory distress and frequent desaturations. The blood gases showed persistent type 2 respiratory failure. A course of intravenous ceftriaxone was commenced as the bronchoalveolar lavage secretion culture grew Enterobacter cloacae. At day 22 of life, a $3^{\text {rd }}$ dose of endotracheal surfactant was given, but no further improvement was noted.

At day 26 of life, the patient was transferred to our center, a tertiary pediatric intensive care unit, for further care. On arrival, he required high ventilatory setting, with oxygenation index of 22. Repeated chest X-ray (Figure 2) showed diffuse bilateral ground-glass opacities (Grade IV), resembling neonatal respiratory distress syndrome. Repeat echocardiogram showed evidence of pulmonary hypertension with acceptable response to inhaled nitric oxide. Subsequent investigations performed included bronchoscopy which showed no malacic airways, bronchoalveolar lavage for cytology which was negative for PAS staining, and serological blood tests which were parvovirus IgM positive and IgG negative, and negative for herpes simplex virus (HSV) IgM, Epstein-Barr virus (EBV) IgM, cytomegalovirus (CMV) and varicella zoster (VZV) IgM. Highresolution computer topography thorax (non-contrasted) (Figure 3) showed diffused ground glass changes in the upper lobes and interstitial thickening; while the middle and lower lobes (especially over left side) showed consolidative changes and reduced lung volume, interlobular septal thickening and bronchial dilatations. Thus, the overall impression was extensive lung damage with fibrosis and scarring. Lung biopsy was not pursued due to difficulties in ventilation and anticipated high risk of complications.

The treatment strategies attempted was as follows: intravenous immunoglobulin $(0.4 \mathrm{~g} / \mathrm{kg} / \mathrm{dose}$ for 2 days), intravenous methylprednisolone pulse therapy $(20 \mathrm{mg} / \mathrm{kg} /$ day, 5 -days courses on monthly basis), hydroxychloroquine $(5 \mathrm{mg} / \mathrm{kg} /$ day $)$ and azithromycin $(7 \mathrm{mg} / \mathrm{kg} /$ dose every other day). However, there was no sustainable improvement noted. Tracheostomy was considered, but parents were not keen for it. Lung transplantation was not a feasible option due to lack of local expertise and technical capacity.

In view of anticipated irrecoverable poor lung function, palliative care was offered and agreed by the parents. At day 83 of life, patient was extubated to high settings of bilevel positive air pressure (BiPAP) noninvasive ventilation (NIV). As there was persistent dependence on high NIV settings with $\mathrm{FiO}_{2}$ requirements of 35 $50 \%$, thus home care was untenable. Life-sustaining support was finally withdrawn when he was 11 months old, and he passed away peacefully in the presence of the family members.

Genetic testing of this patient showed that he had a compound heterozygous variants of c.3364G $>$ A (p. Glu1122Lys) and c. $737 \mathrm{C}>\mathrm{T}$ (p.Pro246Leu) of $A B C A 3$ gene, respectively. The c.3364G $>$ A (p.Glu1122Lys) detected in $A B C A 3$ gene was a missense variant that was not present in dbSNP, clinical and population 
frequency databases. Bioinformatics analysis (Polyphen-2, SIFT and MutationTaster) estimates that this change to have a pathogenic effect. The other variant c.737C > T (p.Pro246Leu) detected in $A B C A 3$ gene was present in dbSNP database (rs144653790) and in population frequency databases gnomAD $(0.00041 \%)$ and the Bioinformatics analysis (Polyphen-2, SIFT and MutationTaster) estimates this change to have a pathogenic effect. This variant had been identified in one infant with neonatal respiratory distress syndrome ${ }^{10}$.

Parental genetic testing revealed that father carried the novel c. 3364G $>$ A (p. Glu1122Lys) mutation, whereas mother carried the c. $737 \mathrm{C}>\mathrm{T}$ (p.Pro246Leu) mutation (Figure 4), thus the variant was in a transposition. Putting this information together, these variants were classified as likely pathogenic based on American College of Medical Genetics and Genomics (ACMG) Standards and Guidelines ${ }^{11}$. Apart from clinching the diagnosis of the patient, the results from the genetics studies facilitated further discussion with parents regarding future family planning and the risks involved.

\section{DISCUSSION}

The clinical presentation of our patient was consistent with a case of neonatal respiratory failure due to surfactant deficiency. Genotype analysis revealed two compound heterozygous variants for $A B C A 3$ [c. 3364G $>\mathrm{A}$ (p. Glu1122Lys) and c. 737C > T (p.Pro246Leu)] in keeping with autosomal recessive mode of inheritance and this is classified as likely pathogenic according to ACMG guidelines.

Cases of $A B C A 3$ mutations leading to severe neonatal respiratory failure has been mostly been reported in the Caucasian population, predominantly in whites, and there was a slight preponderance for the male gender ${ }^{12}$. There were approximately 150 different variants, and most leading to severe neonatal lung disease ${ }^{13}$. This rare condition has been reported in Japan and China with the combined Han and Zhuang populations having carrier rate of $1.3 \%$ of rare $A B C A 3$ mutations which is significantly lower than that in the United States ${ }^{14}$.

Patients with homozygous genotype present with respiratory distress at birth, die or undergo lung transplantation before 1 year of age ${ }^{15}$. In individuals with two $A B C A 3$ mutations, the pulmonary phenotype varies in terms of age of presentation, disease severity, and progression. Children with heterozygous genotypes with respiratory distress at birth may have variable prognosis and outcomes ${ }^{16,17}$. As the criteria of pediatric lung transplantation include individuals at risk of dying from lung failure, pulmonary vascular disease or have a poor quality of life despite maximal medical therapy ${ }^{18}$, lung transplantation was regarded as the fundamental solution for severe chILD ${ }^{19}$, with literature review suggesting a 5-year survival rate of $50 \%^{20}$. However, lung transplantation services in such young children are not readily available due to the high technical expertise required ${ }^{13}$. The role of other commonly attempted therapy such as hydroxychloroquine and corticosteroids remained uncertain ${ }^{21}$. Macrolides were also attempted as it was postulated to inhibit production of proinflammatory cytokines and inflammatory mediators involved in the development of interstitial fibrosis $^{22}$. Other less common therapies include azathioprine, cyclophosphamide, and colchicine ${ }^{23}$.

To the best of our knowledge, this is the first reported patient with child with likely pathogenic compound heterozygous variants in Southeast Asia. The c. 3364G $>$ A (p. Glu1122Lys) was a novel variant that was not previously reported, and this was the first reported patient with both c. 3364G $>$ A (p. Glu1122Lys) and c. $737 \mathrm{C}>\mathrm{T}$ (p.Pro246Leu). We showed in this case study that $A B C A 3$ lung disease can be diagnosed based on clinical evidence of RDS, followed by genetics analysis and imaging without the need for lung biopsy. This was in accordance to both European protocols ${ }^{24}$ and American Thoracic Society Clinical Practice Guideline ${ }^{1}$. In addition to patient diagnosis, genetic testing also provides further direction for future family planning for parents as illustrated in our case. Despite the maximal medical therapy provided, there was no sustainable clinical improvement noted over the course of his 11 months care without lung transplantation, thus reinforcing the need for accurate prognostication for future patients with similar presentations and variants.

In conclusion, newborn patients with unexplained RDS and lung disease should have genetic sequencing for pulmonary surfactant deficiency, mainly for disease prognostication and treatment planning such as tracheostomy and long-term ventilation, lung transplantation or palliative care. Genetic analysis is also 
essential for parental counseling to determine the recurrence risk and provide options of prenatal diagnosis and pre-implantation genetic diagnosis. Hence, it is important to raise awareness of this condition in the Southeast Asia region, so that early diagnosis and prognosis can be made. Further larger population-based studies are required to determine the real frequency of this mutation in our population.

\section{REFERENCE}

(1) Kurland G, Deterding RR, Hagood JS, Young LR, Brody AS, Castile RC, Dell S, Fan LL, Hamvas A, Hilman BC, et al. An Official American Thoracic Society Clinical Practice Guideline: Classification, Evaluation, and Management of Childhood Interstitial Lung Disease in Infancy. Am J Respir Crit Care Med. 2013;1;188(3):376-94.

(2) Hime NJ, Zurynski Y, Fitzgerald D, Selvadurai H, Phu A, Deverell M, Elliott EJ, Jaffe A. Childhood interstitial lung disease: A systematic review. Pediatr Pulmonol. 2015;50(12):1383-92.

(3) Yamano G, Funahashi H, Kawanami O, Zhao LX, Ban N, Uchida Y, Morohoshi T, Ogawa J, Shioda $\mathrm{S}$, Inagaki $\mathrm{N}$. ABCA3 is a lamellar body membrane protein in human lung alveolar type II cells. FEBS Lett. 2001;508(2):221-5.

(4) Mulugeta S, Gray JM, Notarfrancesco KL, Gonzales LW, Koval M, Feinstein S, Ballard PL, Fisher AB, Shuman H. Identification of LBM180, a Lamellar Body Limiting Membrane Protein of Alveolar Type II Cells, as the ABC Transporter Protein ABCA3. J Biol Chem. 2002;277(25):22147-55

(5) Bullard JE, Wert SE, Whitsett JA, Dean M, Nogee LM. ABCA3 Mutations Associated with Pediatric Interstitial Lung Disease. Am J Respir Crit Care Med. 2005;172(8): 1026-1031.

(6) Kuo CS, Young LR. Interstitial Lung Disease in children. Curr Opin Pediatr. 2014;26(3):320-7.

(7) Garmany TH, Moxley MA, White FV, Dean M, Hull WM, Whitsett JA, Nogee LM, Hamvas A. Surfactant Composition and Function in Patients with ABCA3 Mutations. Pediatr Res. 2006;59(6):801-5.

(8) Nogee LM. Genetic Basis of Children's Interstitial Lung Disease. Pediatr Allergy Immunol Pulmonol. 2010;23(1): 15-24.

(9) Shulenin S, Nogee LM, Annilo T, Wert SE, Whitsett JA, Dean M. ABCA3 Gene Mutations in Newborns with Fatal Surfactant Deficiency. N Engl J Med. 2004;350(13):1296-303.

(10) Wambach JA, Wegner DJ, DePass K, Heins H, Druley TD, Mitra RD, An P, Zhang Q, Nogee LM, Cole FS, et al. A single ABCA3 mutations increase risk for neonatal respiratory distress syndrome. Pediatrics. 2012;130(6): e1575-e1582.

(11) Richards S, Aziz N, Bale S, Bick D, Das S, Gastier-Foster J, Grody WW, Hegde M, Lyon E, Spector E, et al. Standards and guidelines for the interpretation of sequence variants: a joint consensus recommendation of the American College of Medical Genetics and Genomics and the Association for Molecular Pathology. Genet Med. 2015;17(5):405-24.

(12) Bullard JE, Wert SE, Whitsett JA, Dean M, Nogee LM. ABCA3 Mutations Associated with Pediatric Interstitial Lung Disease. Am J Respir Crit Care Med. 2005;172(8):1026-1031.

(13) Goncalves JP, Pinheiro L, Costa M, Silva A, Goncalves A, Pereira A. Novel ABCA3 mutations as a cause of respiratory distress in a term newborn. Gene. 2014;534(2):417-20.

(14) Chen YJ, Wambach JA, K DePass, Wegner DJ, Chen S, Zhang Q, Heins H, Cole FS, Hamvas A. Population-based frequency of surfactant dysfunction mutations in a native Chinese cohort. World J Pediatr. 2016;12(2):190-5.

(15) Doan ML, Guillerman RP, Dishop MK, Nogee LM, Langston C, Mallory GB, Sockrider MM, Fan LL. Clinical, radiological and pathological features of ABCA3 mutations in children. Thorax. 2008;63(4):366-73. 
(16) Thavagnanam S, Cutz E, Manson D, Nogee LM, Dell SD. Variable Clinical Outcome of ABCA3 deficiency in two siblings. Pediatr Pulmonol. 2013;48(10):1035-8.

(17) Wambach JA, Casey AM, Fishman MP, Wegner DJ, Wert SE, Cole FS, Hamvas A, Nogee LM. Genotype-Phenotype Correlations for Infants and Children with ABCA3 Deficiency. Am J Respir Crit Care Med. 2014;189(12):1538-43.

(18) Mallory GB, Spray TL. Paediatric Lung Transplantation. Eur Respir J. 2004 Nov;24(5):839-45.

(19) Wei M, Haibo F, Aiqin H, Ma L. A Term Neonatal Case With Lethal Respiratory Failure Associated With a Novel Homozygous Mutation in ABCA3 Gene. Front. Pediatr.2020;8:138.

(20) Mitsiakos G, Tsakalidis C, Karagianni P, Gialamprinou D, Chatziioannidis I, Papoulidis I, Tsanakas I, Soubasi V. A New ABCA3 Gene Mutation c.3445G>A (p.Asp1149Asn) as a Causative Agent of Newborn Lethal Respiratory Distress Syndrome. Medicina (Kaunas). 2019;55(7):389.

(21) Breuer O, Schultz A. Side effects of medications used to treat childhood interstitial lung disease. Paediatr Respir Rev. 2018;28:68-79.

(22) Shinkai M, Henke MO, Rubin BK. Macrolide antibiotics as immunomodulatory medications: proposed mechanisms of action. Pharmacol Ther 2008;117:393-405.

(23) Osika E, Muller M-H, Boccon-Gibod L, Fauroux B, Sardet A, Grosskopf C, Couvreur J, Tournier G, Clement A. Idiopathic pulmonary fibrosis in infants. Pediatr Pulmonol 1997;23:49-54.

(24) Bush A, Cunningham S, de Blic J, Barbato A, Clement A, Epaud R, Hengst M, Kiper N, Nicholson AG, Wetzke M, et al. European protocols for the diagnosis and initial treatment of interstitial lung disease in children. Thorax. 2015;70(11):1078-84.

\section{FIGURES}

Figure 1: Chest X-ray (Day 1 of life) - AP view shows left retrocardiac and upper lobe haziness

Figure 2: Chest X-ray (Day 26 of life) - AP view shows diffuse bilateral ground-glass opacities (Grade IV)

Figure 3: HR-CT Thorax (non-contrast) - Axial and coronal views show ground glass changes and interstitial thickening in upper, middle and lower lobes (especially over left side)

Figure 4: Parental genetics testing results 


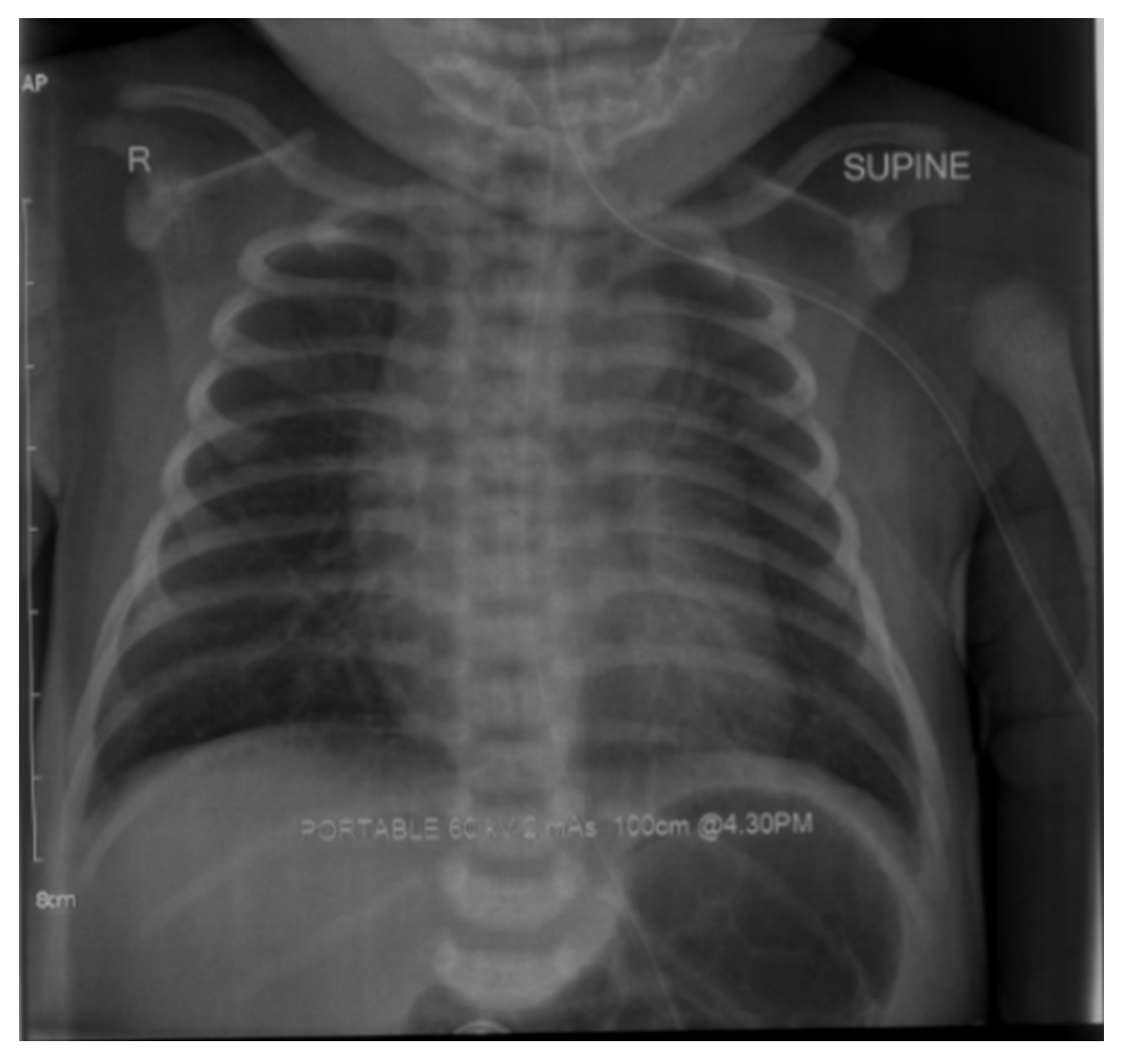



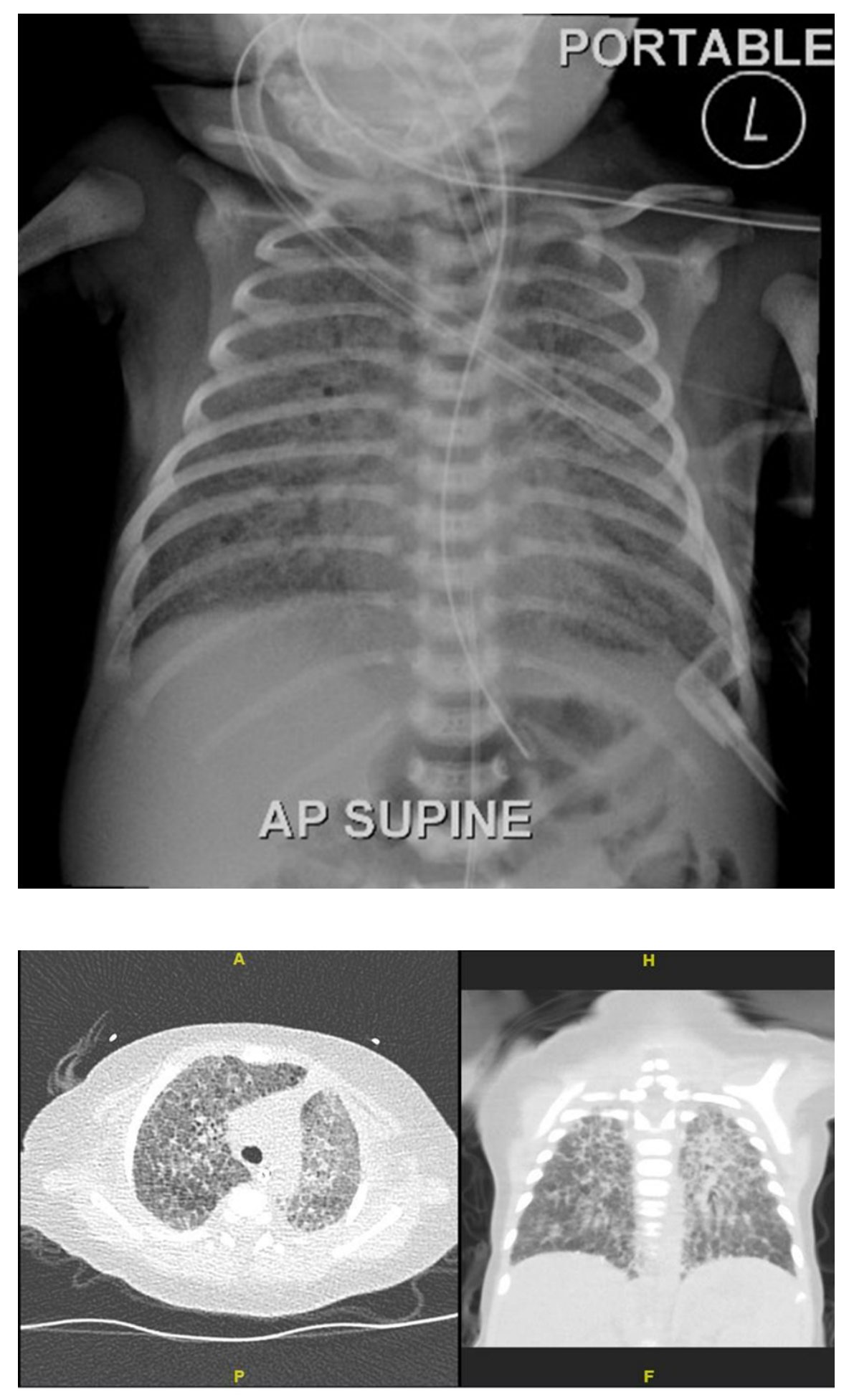
\begin{tabular}{|l|l|}
\hline c.737 C>T, p.(Pro246Leu) in Exon 8 & c.3364 G>A, p.(Glu1122Lys) in Exon 23 \\
\hline
\end{tabular}

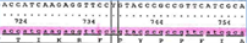

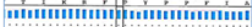

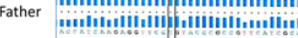

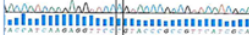

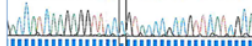

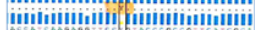

Mother

A heterozygous c.737 C

IIIIIIIIIII IIIIIIIIIIIII mutation detected

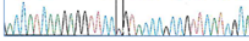

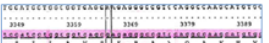

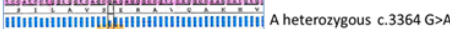

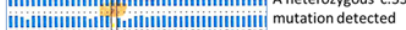

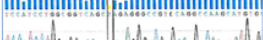

iliI!.!II!IIIIIIIIIIIIInIIIIII!

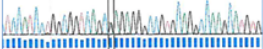

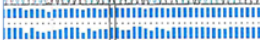

Mother

No mutation detected

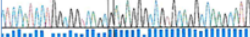

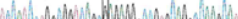

